\title{
Anticipated help seeking behaviour and barriers to seeking care for possible breast and cervical cancer symptoms in Uganda and South Africa
}

\author{
Jennifer Moodley ${ }^{1,2,3 a}$ (D), Deborah Constant ${ }^{1 b}$ (D), Amos Deogratius Mwaka ${ }^{4 c}$ (D), Suzanne Emilie Scott ${ }^{5 d}$ (iD) and Fiona Mary Walter ${ }^{6 e}$ (iD \\ ${ }^{1}$ Women's Health Research Unit, School of Public Health and Family Medicine, Faculty of Health Sciences, University of Cape Town, Observatory, Cape Town, \\ 7925, South Africa \\ ${ }^{2}$ Cancer Research Initiative, Faculty of Health Sciences, University of Cape Town, Observatory, Cape Town, 7925, South Africa \\ ${ }^{3}$ South African Medical Research Council Gynaecology Cancer Research Centre, Faculty of Health Sciences, University of Cape Town, Observatory, Cape Town, \\ 7925, South Africa \\ ${ }^{4}$ Department of Medicine, School of Medicine, College of Health Sciences, Makerere University, Upper Mulago Jill Road PO Box 7072, Kampala, +256, \\ Uganda \\ ${ }^{5}$ Centre for Oral, Clinical and Translational Sciences, Faculty of Dentistry, Oral and Craniofacial Sciences, King's College London, SE1 9RT, UK \\ ${ }^{6}$ The Primary Care Unit, Department of Public Health and Primary Care, University of Cambridge, CB1 8RN, UK \\ ahttps://orcid.org/0000-0002-9398-5202 \\ bhttps://orcid.org/0000-0002-7176-9963 \\ chttps://orcid.org/0000-0001-7952-2327 \\ dhttps://orcid.org/0000-0001-5536-9612 \\ ehttps://orcid.org/0000-0002-7191-6476
}

\section{Abstract}

Objectives: Breast and cervical cancers are leading causes of cancer morbidity and mortality in sub-Saharan Africa. Most women present with advanced-stage disease and have poor outcomes. This study aimed to describe anticipated help-seeking behaviour for possible breast and cervical cancer symptoms, barriers to accessing health care and factors associated with less timely anticipated help-seeking in urban and rural settings in Uganda and South Africa (SA).

Methods: We conducted a cross-sectional community-based survey between August and December 2018. Data were collected from one randomly selected woman per household using the African Women Awareness of CANcer breast and cervical cancer tool. Anticipated help-seeking behaviour was dichotomised into waiting $<1$ week or $\geq 1$ week to seek care. Multivariable analysis identified factors associated with anticipated help-seeking behaviour.

Results: One thousand, seven hundred fifty-eight women participated (Uganda 873, SA 885 , median age 34 , interquartile ranges $26-47$ ). Most would discuss symptoms with someone close to them ( $87.7 \%$ for breast, $83.0 \%$ for cervical symptoms). The majority anticipated seeking care from a health facility in $<1$ week: $86.1 \%$ and $88.0 \%$, respectively, for breast and cervical symptoms. $38.7 \%$ of women expected to encounter at least 1 barrier when seeking care. Lack of money for transport or clinic costs was the most common barrier (24.6\% of participants). For both cancers and in both countries, women who reported more barriers were significantly less likely to anticipate seeking timely care. In $\mathrm{SA}$, rural location was also associated with longer anticipated time to seek care, adjusted prevalence ratio (aPR) 2.92, 95\% confidence interval (CI) 1.48-5.76 and aPR 2.42, 95\% Cl 1.08-5.45 for breast and cervical cancer, respectively.

Correspondence to: Jennifer Moodley Email: Jennifer.moodley@uct.ac.za

ecancer 2021, 15:1171

https://doi.org/10.3332/ecancer.2021.1171

Published: $14 / 01 / 2021$

Received: 03/11/2020

Copyright: ( $)$ the authors; licensee ecancermedicalscience. This is an Open Access article distributed under the terms of the Creative Commons Attribution License (http:// creativecommons.org/licenses/by/3.0), which permits unrestricted use, distribution, and reproduction in any medium, provided the original work is properly cited. 
Conclusion: Interventions that improve community level cancer knowledge and highlight the importance of prompt help-seeking for possible symptoms are important to promote timely care seeking. In addition, addressing financial barriers by reducing transport and clinic costs and tackling geographical inequities in access to care could support women in seeking timely care for possible symptoms.

Keywords: help-seeking, breast cancer, cervical cancer, barriers to care, symptoms

\section{Introduction}

The global burden of cancer is increasing[1]. By 2030, an estimated 24 million people worldwide will develop cancer and 13 million people will die annually from cancer, with $75 \%$ of deaths in low- and middle-income countries (LMICs) [2, 3]. The increasing cancer burden in LMICs is attributed to the high prevalence of risk factors, ageing societies and inequalities in care-seeking, provision and quality of care [4].

Breast and cervical cancers are leading causes of cancer morbidity and mortality among women in LMICs [3]. Cervical cancer accounts for $16 \%$ of the total cancer burden in LMICs, with Africa having the highest age standardised incidence rate (ASIR), 27.6 per 100,000, compared to the world ASIR of 13.1 per 100,000 [3]. Whilst cervical cancer is the leading cause of cancer deaths among women in Africa, breast cancer is the most commonly diagnosed cancer [3]. Although breast cancer incidence rates in Africa are lower than those reported by high-income countries (HICs), mortality rates are similar [3]. For example, ASIRs for breast cancer in UK, South Africa (SA) and Uganda are 94,49 and 21 per 100,000 women, respectively, whilst age-standardised mortality rates are 14, 16 and 10 per 100,000 women, respectively [3].

Stage at diagnosis is an important predictor of cancer outcomes, with advanced stage cancer (stages III or IV) being least responsive to curative treatment. Most African countries do not have cervical or breast cancer screening programmes and the majority of cancers are diagnosed symptomatically and at an advanced stage [4]. In sub-Saharan Africa (SSA), 64\% of breast and $66 \%$ cervical cancers are diagnosed at an advanced stage [5, 6]. By comparison, fewer than a quarter of breast and cervical cancers ( $21 \%$ and $25 \%$, respectively) are diagnosed at an advanced stage in the UK [7]. This contributes to the marked differences in age standardised relative survival (ASRS) between SSA and HICs. Between 2008 and 2015, the 5-year breast cancer survival rate in SSA was 66\% which is similar to the survival rates observed 60 years ago in HICs [5]. Current cervical cancer survival rates in SSA are almost half that seen in the UK (5-year ASRS in SSA 33\% compared to 67\% in UK) [6].

A key goal of comprehensive cancer control is a shift to earlier stage at diagnosis [4]. Minimising the time to diagnosis is dependent on knowledge of relevant symptoms and risk factors; appropriate appraisal of possible cancer symptoms; timely presentation to primary healthcare providers; appropriate assessment at the primary healthcare level and timely access to referral and treatment centres [8, 9]. The current research focuses on the earlier parts of the diagnostic pathway-the decision to seek help. Understanding factors that impact on whether and when symptomatic women seek care can inform efforts to promote earlier diagnosis of cancer, and ultimately better cancer survival. Research in SSA has shown that personal (e.g. knowledge of symptoms, requiring spousal/partner permission to attend health care services), disease (e.g. disease site, symptoms and progression) and healthcare factors (e.g. access to primary health care facilities, facility waiting times) are associated with the timeliness of seeking care [10-13]. However, most of this understanding comes from research undertaken with patients already diagnosed with cancer using small samples and a retrospective design. This has the potential for recall bias, particularly for those who were diagnosed a long time after first noticing symptoms. Little is known about anticipated behaviour prior to a cancer diagnosis, particularly in SSA. Information on anticipated help-seeking behaviour is important to enable the development of effective interventions to reduce delay and promote timeliness of presentation and diagnosis. The aim of this study was to describe women's anticipated help-seeking behaviour for possible breast and cervical cancer symptoms, to describe perceived barriers to accessing health care, and to determine factors associated with less timely anticipated help-seeking in urban and rural settings in Uganda (a low-income country) and SA (a middle-income country). 


\section{Methods}

\section{Study design}

We conducted a community-based cross-sectional survey of women aged 18 years and over in one urban and one rural site in both Uganda and SA (total of four sites). Information on the study sites, sample size, data collection methods and results related to awareness of breast and cervical cancer risk factors, symptoms and lay beliefs have been published elsewhere [10] and are only described briefly here. Methods relevant to the help-seeking behaviour and barriers to care are described in more detail below.

\section{Participants}

At each site, households were selected using systematic random sampling. One woman per identified household was then randomly selected and invited to participate in the study. Women unable to speak either English, Acholi (Uganda) or isiXhosa (SA), and women with a history of breast or cervical cancer, were excluded.

\section{Data collection}

Data were collected by trained interviewers using a hand-held electronic tablet customised with the African Women Awareness of CANcer (AWACAN) tool (Moodley et al AWACAN validation), which is available in English, Acholi and isiXhosa. Women were interviewed face-to-face in their homes, either in their local language (Acholi in Uganda and isiXhosa in SA) or in English depending on their preference.

\section{Measures of socio-demographic variables}

We collected the following socio-demographic details: age, relationship status, educational level, employment status and information on access to assets. We constructed a composite asset index to describe socioeconomic status using principal component analysis on a model that included asset variables with a positive correlation at $p \leq 0.01$. The asset index was then divided into terciles.

\section{Measures of cancer awareness}

\section{Risk factor and symptom awareness}

Risk factor and symptom awareness for both cancers were measured using a closed/prompted question format as laid out in the AWACAN tool: 13 items on breast cancer risk factors, 15 items on breast cancer symptoms and 11 items each on cervical cancer risk factors and symptoms. In addition, the AWACAN tool includes prompted lay belief items: six on breast cancer risk factors and four on cervical cancer risk factors. Lay beliefs refer to locally held beliefs for which there is no current scientific evidence and play an important role in risk interpretation and help-seeking behaviour [10, 11]. Women scored '1' for 'Yes' and '0' for 'No' or 'Don't know'. Scores for each item were summed to form separate knowledge scores for breast cancer risk factors (0-13), breast cancer symptoms (0-15), breast cancer lay beliefs (0-6), cervical cancer risk factors (0-11), cervical cancer symptoms (0-11) and cervical cancer lay beliefs (0-4). Higher scores indicated better recognition of symptoms and risk factors and greater endorsement of lay beliefs.

\section{Confidence in symptom detection}

Confidence in symptom detection was measured by asking respondents 'Are you confident that you would notice a change in your breasts/ symptom that could be cervical cancer ?' (Yes $=1$, No $=0$ ). 


\section{Measure of barriers to seeking care}

To measure barriers, the AWACAN tool stem question asks 'Would any of the following reasons make it difficult for you to see the nurse or clinical officer or doctor if you noticed a symptom or sign which you think may be serious, for example, a change in your breast or a change in the mouth of your womb or cervix that could be cancer?'.[14]. Barriers to seeking care included five personal emotional barriers (e.g. 'worried about what they may find wrong'), four health service barriers (e.g. 'long waiting times at health facility') and three practical barriers (e.g. 'lack of money for transport or facility charges'). Responses were recorded as Agree, Disagree or Don't know. The overall barrier score was the number of 'Agree' responses (range 0-12).

\section{Measures of anticipated help-seeking}

The AWACAN tool has questions relating to anticipated help-seeking behaviours for each cancer separately. These include whether the participant would try self-medication, tell someone close to them, visit a traditional healer and visit a health care facility. For each of these items, participants could respond Yes, No or Don't Know. Anticipated time to seeking care at a health facility was recorded in pre-determined categories: never; $<1$ week; $\geq 1$ week $<1$ month; $\geq 1$ month $<3$ months; $\geq$ months. Responses were then dichotomised into $<1$ week (shorter) versus $\geq 1$ week (longer) to seek care.

\section{Statistical analysis}

We analysed the data using Stata version 15, using valid percentages to report sociodemographic data and anticipated help-seeking responses. Medians and interquartile ranges (IQR) are reported for continuous variables. As Ugandan and South African sociodemographic indicators showed significant differences, we did the bivariate and multivariable analysis by country to best demonstrate these context specific effects. As longer time to seek help ( $\geq 1$ week) occurred in $>10 \%$ for both cancers in each country, we performed Poisson regression with robust variance to identify factors associated with anticipated time to seek help, individually and independently in a multivariable model. Adjusted prevalence ratios (aPR) and 95\% confidence intervals (Cls) are reported. The multivariable Poisson regression models included the following variables: location, age, relationship status, education level, paid work and asset index, risk factor and symptom knowledge scores, risk factor lay beliefs, barriers and confidence in detecting a symptom. Statistical significance was set at the $p<0.05$ level.

\section{Ethics}

Ethics approval for the study was obtained from University of Cape Town, Faculty of Health Sciences Human Research Ethics Committee (HREC 544/2016), the Lacor Hospital Institutional Research Ethics Committee (LHIREC 027/11/2016) and the Ugandan National Council of Science and Technology (HS60ES). Written informed consent was obtained from all participants.

\section{Public involvement}

At the start of the project, community-based project advisory committees (PACs) were set up in each country. The PACs included local government councillors, traditional leaders as well as representatives from the cancer registry and public sector health services (health service providers and managers). The AWACAN tool used in the survey was developed with input from PAC members. In this study, PAC members assisted in gaining access to communities, recruiting local women as field workers in each site and providing guidance on field worker safety. AWACAN results have been discussed with PAC members and field workers in a series of meetings. 


\section{Results}

Between August and December 2018, a total of 1,941 households were visited in Uganda and SA, and 1,758 participants recruited. The overall refusal rate was $6.3 \%$. Details of site recruitment, reasons for refusal and ineligibility and final enrolment numbers have been published previously [10].

\section{Participant profile, cancer awareness and confidence in detecting symptoms}

Participant characteristics by country and site are outlined in Table 1. The overall median age of respondents was 34 years (IQR 26-47). For each country, there were significant urban/rural differences in participants age, relationship status, educational level, work status and asset index profile. A higher proportion of women in rural Uganda were married and had no or incomplete primary schooling compared to other sites. Overall, $66.3 \%$ of participants did no paid work, with the highest rate in rural SA (91.8\%). Results on awareness of breast and cervical cancer symptoms, risk factors and lay beliefs are described elsewhere [10] and summary results included in Table 1. Most women (88.3\%) were confident that they would notice a breast change. Overall $66.5 \%$ of women reported that they were confident in noticing cervical symptoms, with differences in confidence levels between countries and between urban and rural settings.

Table 1. Participant profile.

\begin{tabular}{|c|c|c|c|c|c|c|c|}
\hline & \multicolumn{3}{|c|}{ SA } & \multicolumn{3}{|c|}{ Uganda } & \multirow{3}{*}{$\begin{array}{c}\text { Total } \\
n(\%) \\
n=1758 \\
\end{array}$} \\
\hline & Urban & Rural & \multirow[b]{2}{*}{$p$-value ${ }^{a}$} & Urban & Rural & \multirow[b]{2}{*}{$p$-value ${ }^{a}$} & \\
\hline & $\begin{array}{c}n(\%) \\
n=445\end{array}$ & $\begin{array}{c}n(\%) \\
n=428\end{array}$ & & $\begin{array}{c}n(\%) \\
n=458\end{array}$ & $\begin{array}{c}n(\%) \\
n=427\end{array}$ & & \\
\hline \multicolumn{8}{|l|}{ Age (years) } \\
\hline $18-29$ & $130(30.4)$ & $106(25.2)$ & \multirow[t]{4}{*}{$<0.001$} & $216(47.2)$ & $186(44.0)$ & \multirow[t]{4}{*}{0.04} & $638(36.9)$ \\
\hline $30-49$ & $221(51.6)$ & $170(40.4)$ & & $174(38.0)$ & $146(34.5)$ & & 711 (41.1) \\
\hline$\geq 50$ & 77 (18.0) & $145(34.4)$ & & $68(14.9)$ & $91(21.5)$ & & $381(22.0)$ \\
\hline Median (IQR) & $36(28-45)$ & $40(29-54)$ & & $30(24-40)$ & $32(23-46)$ & & $34(26-47)$ \\
\hline \multicolumn{8}{|l|}{ Relationship status } \\
\hline $\begin{array}{l}\text { Married/living with a } \\
\text { partner }\end{array}$ & $185(41.8)$ & $200(46.7)$ & \multirow[t]{3}{*}{0.01} & $300(65.5)$ & $314(73.5)$ & \multirow[t]{3}{*}{0.01} & $999(56.9)$ \\
\hline $\begin{array}{l}\text { No partner/not living } \\
\text { with partner }\end{array}$ & $224(50.6)$ & $174(40.7)$ & & $36(7.9)$ & $17(4.0)$ & & $451(25.7)$ \\
\hline $\begin{array}{l}\text { Separated/divorced/ } \\
\text { widowed }\end{array}$ & $34(7.7)$ & $54(12.6)$ & & $122(26.6)$ & $96(22.5)$ & & $306(17.4)$ \\
\hline \multicolumn{8}{|l|}{ Highest educational level } \\
\hline $\begin{array}{l}\text { No schooling and primary } \\
\text { incomplete }\end{array}$ & $22(5.0)$ & $162(38.0)$ & \multirow[t]{3}{*}{$<0.001$} & $171(37.3)$ & $328(76.8)$ & \multirow[t]{3}{*}{$<0.001$} & $683(39.0)$ \\
\hline $\begin{array}{l}\text { Primary complete and } \\
\text { secondary incomplete }\end{array}$ & $196(44.3)$ & $178(41.8)$ & & $167(36.5)$ & $84(19.7)$ & & $625(35.6)$ \\
\hline $\begin{array}{l}\text { Secondary complete or } \\
\text { more }\end{array}$ & $224(50.7)$ & $86(20.2)$ & & $120(26.2)$ & $15(3.5)$ & & $445(25.4)$ \\
\hline \multicolumn{8}{|l|}{ Paid work } \\
\hline Yes & $226(51.6)$ & $35(8.2)$ & \multirow[t]{2}{*}{$<0.001$} & $217(47.6)$ & $110(25.8)$ & \multirow[t]{2}{*}{$<0.001$} & $588(33.7)$ \\
\hline No & $212(48.4)$ & 391 (91.8) & & $239(52.4)$ & $317(74.2)$ & & 1159 (66.3) \\
\hline
\end{tabular}


Table 1. Participant profile. (Continued)

\begin{tabular}{|c|c|c|c|c|c|c|c|}
\hline \multicolumn{8}{|l|}{ Asset index } \\
\hline Upper tercile & $326(73.3)$ & $6(1.4)$ & \multirow[t]{3}{*}{$<0.001$} & $253(55.2)$ & $70(16.4)$ & \multirow[t]{3}{*}{$<0.001$} & $655(37.3)$ \\
\hline Middle tercile & 79 (17.8) & $182(42.5)$ & & $122(26.6)$ & $210(49.2)$ & & $593(33.7)$ \\
\hline Lower tercile & $40(9.0)$ & $240(56.1)$ & & $83(18.1)$ & $147(34.4)$ & & $510(29.0)$ \\
\hline \multicolumn{8}{|l|}{ Breast cancer awareness } \\
\hline $\begin{array}{l}\text { Median (IQR) risk factor } \\
\text { awareness score }\end{array}$ & $5(3-9)$ & $4(2-6)$ & $<0.001$ & $5(2-7)$ & $5(3-7)$ & 0.38 & $4(3-7)$ \\
\hline $\begin{array}{l}\text { Median (IQR) symptom } \\
\text { awareness score }\end{array}$ & $15(14-15)$ & $13(10-14)$ & $<0.001$ & $13(11-15)$ & $13(10-15)$ & 0.24 & $14(11-15)$ \\
\hline $\begin{array}{l}\text { Median (IQR) number of } \\
\text { lay beliefs endorsed }\end{array}$ & $5(4-5)$ & $4(2-5)$ & $<0.001$ & $4(4--5)$ & $5(3-5)$ & 0.82 & $4(4-5)$ \\
\hline \multicolumn{8}{|l|}{ Cervical cancer awareness } \\
\hline $\begin{array}{l}\text { Median (IQR) risk factor } \\
\text { awareness score }\end{array}$ & $9(7-10)$ & $8(6-9)$ & $<0.001$ & $7(5-9)$ & $8(6-9)$ & 0.01 & $8(6-9)$ \\
\hline $\begin{array}{l}\text { Median (IQR) symptom } \\
\text { awareness score }\end{array}$ & $9(8-11)$ & $6(8-10)$ & $<0.001$ & $9(7-10)$ & $9(6-10)$ & 0.90 & $9(7-10)$ \\
\hline $\begin{array}{l}\text { Median (IQR) number of } \\
\text { lay beliefs endorsed }\end{array}$ & $2(2-3)$ & $2(2-3)$ & $<0.001$ & $2(2-3)$ & $2(2-3)$ & 0.46 & $2(2-3)$ \\
\hline $\begin{array}{l}\text { Confident in noticing a } \\
\text { breast change }\end{array}$ & $383(86.1)$ & $403(94.2)$ & $<0.001$ & $410(89.5)$ & $357(83.6)$ & 0.01 & $1553(88.3)$ \\
\hline $\begin{array}{l}\text { Confident in noticing a } \\
\text { cervical symptom }\end{array}$ & 371 (83.4) & 333 (77.8) & 0.04 & $274(59.8)$ & $191(44.7)$ & $<0.001$ & $1169(66.5)$ \\
\hline
\end{tabular}

There were missing data for: Age: SA urban; $n=17$, Rural; $n=7$. Uganda rural; $n=4$. Total; $n=28$; Relationship status: SA urban; $n=2$. Total; $n=2 ;$ Highest educational level: SA urban; $n=3$, Rural; $n=2$. Total; $n=5$; Paid work: SA urban; $n=7$, Rural; $n=2$. Uganda urban $=2$. Total; $n=11$

aChi squared tests for proportions and Wilcoxon Signed Rank Test for medians

$\mathrm{IQR}$, Interquartile range

\section{Barriers to seeking medical help}

Overall, $38.7 \%$ of women reported at least one barrier to seeking medical care: $15.3 \%$ experienced at least one emotional barrier, $22.7 \%$ at least one health service barrier and $27.5 \%$ at least one practical barrier. Table 2 provides information on the emotional, practical and health system barriers to accessing care for possible breast and cervical cancer symptoms by each site. Overall the most common barrier reported was financial, i.e. not having money for transport or clinic costs, which was reported by $24.6 \%$ of all respondents. In SA, significantly more rural compared to urban women experienced a financial barrier (18.7\% versus $6.5 \%$. $p<0.01)$. In Uganda, a high and similar proportion of rural women and urban women reported a financial barrier (38.2\% and $36.2 \%$, respectively, $p=0.35$ ).

\section{Anticipated response to possible breast and cervical cancer symptoms}

Most women reported that they would discuss possible symptoms with someone close to them $(87.7 \%$ for breast, $83.0 \%$ for cervical symptoms), with significant differences between urban and rural participants in SA (see Table 3). A quarter of women would try self-medication for breast symptoms and a fifth for cervical symptoms, with rural women significantly more likely to try self-medication for both cancers compared to urban women. Few women reported that they would visit a traditional healer $(9.3 \%$ for breast and $8.1 \%$ for cervical-Table 3 provides details by each site). Of those that would visit a traditional healer for a symptom, just over half would do this in less than 1 week. 
Table 2. Barriers to help seeking for breast and cervical cancers.

\begin{tabular}{|c|c|c|c|c|c|c|c|}
\hline & \multicolumn{3}{|c|}{ SA } & \multicolumn{3}{|c|}{ Uganda } & \multirow{3}{*}{$\begin{array}{c}\text { Total } \\
\\
n(\%) \\
n=1,758\end{array}$} \\
\hline & Urban & Rural & \multirow[b]{2}{*}{$p$-value ${ }^{b}$} & Urban & Rural & \multirow[b]{2}{*}{$p$-value ${ }^{b}$} & \\
\hline & $\begin{array}{c}n(\%) \\
n=445\end{array}$ & $\begin{array}{c}n(\%) \\
n=428\end{array}$ & & $\begin{array}{c}n(\%) \\
n=458\end{array}$ & $\begin{array}{c}n(\%) \\
n=427\end{array}$ & & \\
\hline \multicolumn{8}{|l|}{ Personal emotional barriers } \\
\hline $\begin{array}{l}\text { Worried about tests that } \\
\text { may be done }\end{array}$ & $33(7.4)$ & $34(7.9)$ & 0.77 & $37(8.1)$ & $24(5.6)$ & 0.15 & $128(7.3)$ \\
\hline $\begin{array}{l}\text { Worried about what they } \\
\text { might find wrong }\end{array}$ & $25(5.6)$ & $20(4.7)$ & 0.53 & $27(5.9)$ & $30(7.0)$ & 0.49 & $102(5.8)$ \\
\hline Feel embarrassed & $25(5.6)$ & $26(6.1)$ & 0.77 & $27(5.9)$ & $33(7.7)$ & 0.28 & $111(6.3)$ \\
\hline $\begin{array}{l}\text { Not confident to talk } \\
\text { about symptoms with the } \\
\text { doctor }\end{array}$ & $23(5.2)$ & $27(6.3)$ & 0.47 & $28(6.1)$ & $37(8.7)$ & 0.15 & $115(6.3)$ \\
\hline $\begin{array}{l}\text { Worried about wasting } \\
\text { doctor's time }\end{array}$ & $20(4.5)$ & $21(4.9)$ & 0.77 & $6(1.3)$ & $9(2.1)$ & 0.36 & $56(3.2)$ \\
\hline $\begin{array}{l}\text { Fatalistic cancer belief-it } \\
\text { is a serious and will die } \\
\text { anyway }\end{array}$ & $2(0.5)$ & $12(2.8)$ & 0.01 & $17(3.7)$ & $26(6.1)$ & 0.10 & $57(3.2)$ \\
\hline \multicolumn{8}{|l|}{ Personal practical barriers } \\
\hline $\begin{array}{l}\text { Lack of money for trans- } \\
\text { port or facility charges }\end{array}$ & $29(6.5)$ & 80 (18.7) & $<0.001$ & $161(35.2)$ & $163(38.2)$ & 0.35 & $433(24.6)$ \\
\hline Other priorities & $11(2.5)$ & $20(4.7)$ & 0.08 & $25(5.5)$ & $45(10.5)$ & 0.01 & $101(5.8)$ \\
\hline $\begin{array}{l}\text { Husband/partner would } \\
\text { not allow me to go }\end{array}$ & $7(1.6)$ & $10(2.3)$ & 0.41 & $35(8.2)$ & $18(3.9)$ & 0.01 & $70(4.0)$ \\
\hline \multicolumn{8}{|l|}{ Health system barriers } \\
\hline $\begin{array}{l}\text { Long health facility wait- } \\
\text { ing times }\end{array}$ & $83(18.7)$ & $62(14.5)$ & 0.10 & $48(10.5)$ & $41(9.6)$ & 0.66 & $234(13.3)$ \\
\hline $\begin{array}{l}\text { Past bad experience at } \\
\text { health facility }\end{array}$ & $35(7.9)$ & $27(6.3)$ & 0.37 & $47(10.3)$ & $34(8.0)$ & 0.24 & $143(8.1)$ \\
\hline $\begin{array}{l}\text { Health practitioner might } \\
\text { not understand my lan- } \\
\text { guage or culture }\end{array}$ & $8(1.8)$ & $23(5.4)$ & 0.01 & $57(12.5)$ & $52(12.2)$ & 0.90 & $140(8.0)$ \\
\hline
\end{tabular}

${ }^{a} \mathrm{~N}=1,757$ (missing data for one woman in rural SA)

${ }^{\mathrm{b}}$ Chi squared tests for proportions and Wilcoxon Signed Rank Test for medians

The majority of women anticipated that they would visit a healthcare facility within 1 week of noticing a breast symptom (86.1\%) or cervical symptom (88.0\%) (Table 3).

\section{Factors associated with longer anticipated time to seek healthcare}

\section{(a) for possible symptoms of breast cancer}

Associations between independent factors and longer anticipated help-seeking for possible symptoms of breast cancer are presented in Table 4. Multivariable analyses indicated that in SA, significant determinants of longer anticipated time to seek healthcare were rural 
compared to urban location (aPR 2.92, 95\% Cl 1.48-5.76), experiencing more barriers (aPR 1.18,95\% Cl 1.08-1.29), lower risk factor knowledge (aPR 0.91, 95\% Cl 0.83-0.99) and having more assets (aPR 0.27, 95\% Cl 0.13-0.55, aPR 0.37, 95\% Cl 0.17-0.79 for middle and lower terciles compared to upper tercile, respectively). In Uganda, factors significantly associated with longer anticipated time to seek healthcare were higher risk factor knowledge (aPR 1.07, 95\% Cl 1.01-1.13), experiencing more barriers to care (aPR 1.33, 95\% $\mathrm{Cl} 1.26-1.40)$ and endorsing fewer lay beliefs (aPR 0.87, 95\% $\mathrm{Cl} 0.78-0.97)$.

Table 3. Anticipated responses to breast cancer and cervical cancer symptoms.

\begin{tabular}{|c|c|c|c|c|c|c|c|}
\hline & \multicolumn{3}{|c|}{ SA } & \multicolumn{3}{|c|}{ Uganda } & \multirow{3}{*}{$\begin{array}{c}\text { Total } \\
n(\%) \\
n=1,758\end{array}$} \\
\hline & Urban & Rural & \multirow[b]{2}{*}{$p$-value ${ }^{a}$} & Urban & Rural & \multirow[b]{2}{*}{$p$-value ${ }^{a}$} & \\
\hline & $\begin{array}{c}n(\%) \\
n=445\end{array}$ & $\begin{array}{c}n(\%) \\
n=428\end{array}$ & & $\begin{array}{c}n(\%) \\
n=458\end{array}$ & $\begin{array}{c}n(\%) \\
n=427\end{array}$ & & \\
\hline \multicolumn{8}{|c|}{ Response to noticing a breast change } \\
\hline Try self-medication & $110(24.7)$ & $157(36.7)$ & $<0.001$ & $76(16.6)$ & $106(24.8)$ & $<0.01$ & $449(25.5)$ \\
\hline $\begin{array}{l}\text { Tell someone close } \\
\text { to you }\end{array}$ & $359(80.7)$ & $405(94.6)$ & $<0.001$ & $395(86.2)$ & $382(89.5)$ & 0.14 & $1,541(87.7)$ \\
\hline \multicolumn{8}{|l|}{$\begin{array}{l}\text { Visit the healthcare } \\
\text { facility in: }\end{array}$} \\
\hline$<1$ week & $403(90.6)$ & $378(88.3)$ & 0.28 & $380(83.0)$ & $353(82.7)$ & 0.91 & $1,514(86.1)$ \\
\hline 1 week or more & $42(9.4)$ & $50(11.7)$ & & $78(17.0)$ & $74(17.3)$ & & $244(13.9)$ \\
\hline $\begin{array}{l}\text { Visit a traditional } \\
\text { healer }\end{array}$ & $53(11.9)$ & $37(8.64)$ & 0.11 & $32(7.0)$ & $41(9.6)$ & 0.16 & $163(9.3)$ \\
\hline $\begin{array}{l}\text { Time to visit a } \\
\text { traditional healer }\end{array}$ & $n=51$ & $n=35$ & & $n=32$ & $n=41$ & & $n=159$ \\
\hline$<1$ week & $34(66.7)$ & $27(77.1)$ & 0.29 & $9(28.1)$ & $17(41.5)$ & 0.24 & $87(54.7)$ \\
\hline 1 week or more & $17(33.3)$ & $8(22.9)$ & & $23(71.9)$ & $24(58.5)$ & & $72(45.3)$ \\
\hline \multicolumn{8}{|c|}{ Response to noticing a cervical symptom } \\
\hline Try self-medication & $90(20.2)$ & $145(33.9)$ & $<0.001$ & $62(13.5)$ & $83(19.4)$ & 0.02 & $380(21.6)$ \\
\hline $\begin{array}{l}\text { Tell someone close } \\
\text { to you }\end{array}$ & 351 (78.9) & $403(94.4)$ & $<0.001$ & $362(79.0)$ & $342(80.1)$ & 0.70 & $1458(83.0)$ \\
\hline \multicolumn{8}{|l|}{$\begin{array}{l}\text { Visit the healthcare } \\
\text { facility in: }\end{array}$} \\
\hline$<1$ week & $406(91.2)$ & $384(89.7)$ & 0.45 & $403(88.0)$ & $355(83.1)$ & 0.04 & $1548(88.0)$ \\
\hline 1 week or more & $39(8.8)$ & $44(10.3)$ & & $55(12.0)$ & $72(16.9)$ & & $210(12.0)$ \\
\hline $\begin{array}{l}\text { Visit a traditional } \\
\text { healer }\end{array}$ & $43(9.7)$ & $34(8.0)$ & 0.38 & $28(6.1)$ & $37(8.7)$ & 0.15 & $142(8.1)$ \\
\hline $\begin{array}{l}\text { Time to visit a } \\
\text { traditional healer }\end{array}$ & $n=43$ & $n=33$ & & $n=25$ & $n=37$ & & $n=138$ \\
\hline$<1$ week & $29(67.4)$ & $22(6.7)$ & 0.94 & $10(40.0)$ & $14(37.8)$ & 0.86 & $75(54.4)$ \\
\hline 1 week or more & $14(32.6)$ & $11(33.3)$ & & $15(60.0)$ & $23(62.2)$ & & $63(45.7)$ \\
\hline
\end{tabular}

There were missing data for Time to visit a traditional healer: SA rural $n=1$; Uganda urban $n=3$

${ }^{a}$ Chi squared tests for proportions and Wilcoxon Signed Rank Test for medians 
Table 4. Factors associated with longer anticipated time to seek care for possible symptoms of breast cancer.

\begin{tabular}{|c|c|c|c|c|c|c|c|c|}
\hline & \multicolumn{4}{|c|}{ SA } & \multicolumn{4}{|c|}{ Uganda } \\
\hline & $\begin{array}{c}\text { Prevalence } \\
\text { ratio (PR) }\end{array}$ & $95 \% \mathrm{CI} P R$ & Adj PR & $\begin{array}{c}95 \% \text { Cl Adj } \\
\text { PR }\end{array}$ & PR & $95 \% \mathrm{CI} P R$ & Adj PR & $\begin{array}{c}95 \% \mathrm{Cl} \text { Adj } \\
\text { PR }\end{array}$ \\
\hline \multicolumn{9}{|l|}{ Location } \\
\hline Urban & Referent & & Referent & & Referent & & Referent & \\
\hline Rural & 1.24 & $0.84-1.83$ & $2.92^{\mathrm{a}}$ & $1.48-5.76$ & 1.01 & $0.76-1.36$ & 0.89 & $0.63-1.24$ \\
\hline \multicolumn{9}{|l|}{ Age } \\
\hline $50+$ & Referent & & & & & & & \\
\hline $30 £ 50$ & 1.46 & $0.81-2.65$ & 1.78 & $0.82-3.87$ & 1.05 & $0.70-1.57$ & 0.88 & $0.56-1.38$ \\
\hline $18<30$ & $2.49^{a}$ & $1.38-4.47$ & 1.72 & $0.77-3.85$ & 0.92 & $0.61-1.37$ & 0.94 & $0.59-1.51$ \\
\hline \multicolumn{9}{|l|}{ Relationship status } \\
\hline Married/living with a partner & Referent & & & & & & & \\
\hline Single & $1.64^{\mathrm{a}}$ & $1.09-2.47$ & 1.26 & $0.77-2.06$ & 1.32 & $0.78-2.24$ & 1.32 & $0.76-2.27$ \\
\hline Widowed/separated/divorced & 0.40 & $0.12-1.27$ & 0.59 & $0.17-2.02$ & 0.94 & $0.66-1.33$ & 0.82 & $0.55-1.23$ \\
\hline \multicolumn{9}{|l|}{ Highest educational level } \\
\hline Secondary complete or more & Referent & & & & & & & \\
\hline Primary complete to secondary incomplete & 1.24 & $0.80-1.92$ & 1.27 & $0.78-2.09$ & 1.28 & $0.77-2.14$ & 1.32 & $0.80-2.18$ \\
\hline No schooling to primary incomplete & 0.84 & $0.47-1.52$ & 0.91 & $0.40-2.09$ & 1.37 & $0.86-2.19$ & 1.34 & $0.76-2.38$ \\
\hline \multicolumn{9}{|l|}{ Paid work } \\
\hline Yes & Referent & & & & & & & \\
\hline No & 1.21 & $0.78-1.88$ & 1.00 & $0.56-1.76$ & 1.00 & $0.74-1.35$ & 1.06 & $0.78-1.47$ \\
\hline \multicolumn{9}{|l|}{ Asset index } \\
\hline Upper tercile & Referent & & & & & & & \\
\hline Middle tercile & $0.53^{a}$ & $0.31-0.91$ & $0.27^{a}$ & $0.13-0.55$ & 1.06 & $0.76-1.48$ & 0.88 & $0.58-1.34$ \\
\hline Lower tercile & 0.98 & $0.64-1.51$ & $0.37^{a}$ & $0.17-0.79$ & 0.94 & $0.64-1.38$ & 0.80 & $0.50-1.28$ \\
\hline Breast cancer risk factor knowledge score & $0.88^{\mathrm{a}}$ & $0.83-0.95$ & $0.91^{\mathrm{a}}$ & $0.83-0.99$ & 0.98 & $0.93-1.03$ & $1.07^{\mathrm{a}}$ & $1.01-1.13$ \\
\hline Breast cancer symptom knowledge score & $0.94^{a}$ & $0.90-0.99$ & 0.98 & $0.90-1.07$ & $0.95^{\mathrm{a}}$ & $0.91-0.98$ & 0.97 & $0.92-1.02$ \\
\hline $\begin{array}{l}\text { Breast cancer risk factor lay beliefs } \\
\text { endorsed }\end{array}$ & $0.85^{\mathrm{a}}$ & $0.75-0.97$ & 1.00 & $0.83-1.20$ & $0.89^{a}$ & $0.81-0.98$ & $0.87^{a}$ & $0.78-0.97$ \\
\hline Barriers experienced & $1.26^{a}$ & $1.18-1.35$ & $1.18^{\mathrm{a}}$ & $1.08-1.29$ & $1.30^{\mathrm{a}}$ & $1.25-1.36$ & $1.33^{a}$ & $1.26-1.40$ \\
\hline \multicolumn{9}{|l|}{ Confident would notice change in breast/s } \\
\hline Yes & Referent & & & & & & & \\
\hline No & $2.20^{a}$ & $1.38-3.50$ & 1.42 & $0.78-2.61$ & $1.87^{a}$ & $1.35-2.60$ & 1.14 & $0.78-1.65$ \\
\hline
\end{tabular}

${ }^{a} p<0.05$ 


\section{(b) for possible symptoms of cervical cancer}

Factors associated with longer anticipated help-seeking for possible symptoms of cervical cancer are presented in Table 5. As with possible breast cancer symptoms, the multivariable analyses indicated that in SA, women living in rural areas, those experiencing more barriers and those with more assets (aPR 0.34,95\% Cl 0.15-0.80) were more likely to indicate they would wait longer before seeking help (aPR 2.42, 95\% $\mathrm{Cl} 1.08-5.45$ and aPR 1.19, 95\% Cl 1.06-1.34, respectively). Among Ugandan women, experiencing more barriers to accessing care was significantly associated with less timely anticipated help-seeking (aPR 1.23, 95\% Cl 1.16-1.30).

Table 5. Factors associated with longer anticipated time to seek care for possible symptoms of cervical cancer.

\begin{tabular}{|c|c|c|c|c|c|c|c|c|}
\hline & \multicolumn{4}{|c|}{ SA } & \multicolumn{4}{|c|}{ Uganda } \\
\hline & $\begin{array}{l}\text { Prevalence } \\
\text { ratio (PR) }\end{array}$ & $95 \% \mathrm{CI} P R$ & Adj PR & $\begin{array}{l}\text { 95\% Cl Adj } \\
\text { PR }\end{array}$ & PR & $95 \% \mathrm{Cl} P R$ & Adj PR & $\begin{array}{l}\text { 95\% Cl Adj } \\
\text { PR }\end{array}$ \\
\hline \multicolumn{9}{|l|}{ Location } \\
\hline Urban & Referent & & Referent & & Referent & & Referent & \\
\hline Rural & 1.17 & $0.77-1.77$ & $2.42^{\mathrm{a}}$ & $1.08-5.45$ & $1.40^{a}$ & $1.01-1.94$ & 1.23 & $0.84-1.78$ \\
\hline \multicolumn{9}{|l|}{ Age } \\
\hline $50+$ & Referent & & & & & & & \\
\hline $30 £ 50$ & 0.88 & $0.51-1.51$ & 0.79 & $0.43-1.43$ & 0.76 & $0.50-1.16$ & 0.79 & $0.49-1.27$ \\
\hline $18<30$ & 1.32 & $0.76-2.27$ & 0.74 & $0.36-1.52$ & 0.67 & $0.45-1.12$ & 0.78 & $0.47-1.29$ \\
\hline \multicolumn{9}{|l|}{ Relationship status } \\
\hline Married/living with a partner & Referent & & & & & & & \\
\hline Single & $1.55^{\mathrm{a}}$ & $1.00-2.39$ & 1.26 & $0.74-2.13$ & 1.14 & $0.59-2.24$ & 1.37 & $0.74-2.52$ \\
\hline Widowed/separated/divorced & 0.73 & $0.29-1.83$ & 0.64 & $0.22-1.87$ & 1.32 & $0.93-1.88$ & 1.04 & $0.67-1.61$ \\
\hline \multicolumn{9}{|l|}{ Highest educational level } \\
\hline Secondary complete or more & Referent & & & & & & & \\
\hline Primary complete to secondary incomplete & 1.49 & $0.92-2.39$ & 1.48 & $0.85-2.58$ & 1.37 & $0.70-2.66$ & 1.24 & $0.64-2.40$ \\
\hline No schooling to primary incomplete & 1.05 & $0.56-1.96$ & 1.09 & $0.47-2.53$ & $2.16^{a}$ & $1.19-3.93$ & 1.73 & $0.85-3.53$ \\
\hline \multicolumn{9}{|l|}{ Paid work } \\
\hline Yes & Referent & & & & & & & \\
\hline No & 0.90 & $0.58-1.39$ & 0.62 & $0.36-1.06$ & 0.92 & $0.66-1.29$ & 0.84 & $0.60-1.19$ \\
\hline \multicolumn{9}{|l|}{ Asset index } \\
\hline Upper tercile & Referent & & & & & & & \\
\hline Middle tercile & 0.60 & $0.34-1.06$ & $0.34^{a}$ & $0.15-0.80$ & 1.30 & $0.88-1.91$ & 0.92 & $0.59-1.44$ \\
\hline Lower tercile & 1.15 & $0.73-1.81$ & 0.64 & $0.27-1.55$ & 1.30 & $0.85-1.97$ & 0.82 & $0.50-1.36$ \\
\hline Cervical cancer risk factor knowledge score & 0.95 & $0.87-1.03$ & 0.97 & $0.87-1.08$ & $0.91^{a}$ & $0.86-0.97$ & 0.94 & 0.87-1.01 \\
\hline Cervical cancer symptom knowledge & 0.94 & $0.88-1.01$ & 0.97 & $0.86-1.10$ & $0.90^{a}$ & $0.86-0.95$ & 0.96 & $0.90-1.03$ \\
\hline $\begin{array}{l}\text { Cervical cancer risk factor lay beliefs } \\
\text { endorsed }\end{array}$ & 1.02 & $0.80-1.29$ & 1.21 & $0.93-1.56$ & $1.03^{\mathrm{a}}$ & $0.85-1.23$ & 1.19 & $0.96-1.47$ \\
\hline Barriers experienced & $1.23^{a}$ & $1.13-1.34$ & $1.19^{a}$ & $1.06-1.34$ & $1.28^{a}$ & $1.22-1.34$ & $1.23^{a}$ & $1.16-1.30$ \\
\hline \multicolumn{9}{|c|}{ Confident would notice a symptom that could be cervical cancer } \\
\hline \multicolumn{9}{|l|}{ Yes } \\
\hline No & $1.60^{\mathrm{a}}$ & $1.02-2.51$ & 1.28 & $0.75-2.18$ & $1.76^{\mathrm{a}}$ & $1.26-2.46$ & 1.24 & $0.86-1.80$ \\
\hline
\end{tabular}

${ }^{a} p<0.05$ 


\section{Discussion}

Prompt help-seeking when a possible symptom is discovered is an important step towards timely cancer diagnosis [4]. This is the first community-based study in SSA reporting on anticipated help-seeking behaviour and barriers to care for possible breast and cervical cancer symptoms. We found that most women did not anticipate delay in seeking care-86\% and $88 \%$ would seek care at a health facility within 1 week for a possible breast or cervical symptom, respectively. This contrasts starkly with actual patient intervals (time between noticing a symptom and presenting to a health facility) reported in LMIC settings [11, 15-17]. For example, for breast cancer, Rayne et al [15] and Moodley et al [17] reported that only $33 \%$ and $25 \%$, respectively, of breast cancer patients attended a health facility within 1 week of noticing a symptom. This discrepancy between hypothetical and actual help-seeking has also been reported in HIC settings [18]. A review of studies measuring time to seeking care for a number of different cancers found that most people anticipated seeking care within 1 week, whereas most patients actually waited for more than 2 months before seeking medical care [18]. This is likely due to the bulk of delay in seeking help arising in the appraisal interval, whereby symptoms are misinterpreted as minor, manageable conditions that do not require attention from a healthcare professional [19]. In this study, we studied the help-seeking interval [8, 9] and have demonstrated that even when women do consider cancer (or something serious) a proportion of women would still not seek help straightway and a number of barriers to healthcare use are key in explaining this delay. Thus, whilst improving cancer knowledge is important, our findings suggest that to improve timely diagnosis, interventions must be directed at more than just improving symptom knowledge and include support to overcome emotional, health service and practical barriers to seeking help.

In our study, almost $40 \%$ of women expected to encounter at least one barrier when seeking care for a possible breast or cervical cancer symptom. Importantly, women who reported more barriers were significantly less likely to seek timely care in both Uganda and SA.Both the Alma-Ata Declaration of 1978 and the Astana Declaration of 2018 identify access to basic health care as a cornerstone to achieving health for all $[20,21]$. In pursuit of this, many countries have committed to strengthening primary health care. Yet for many people, access to care for possible cancer symptoms remains a challenging process of negotiating a series of barriers [22-26]. Identifying these barriers is a key first step that can inform ways to mitigate their impact and foster timely presentation to health services.

As in other LMIC contexts, transportation and facility costs emerged as key access barriers in our study, especially for rural residents [23, $26,27]$. Research has shown that experiencing financial difficulty delays symptomatic presentation and is associated with advanced stage at cancer diagnosis $[11,18,28]$. In countries such as SA where user fees for primary health services have been abolished, there are still transport-related costs borne by individuals. This is a particular burden where health facilities are often far from people's homes. Our finding of a strong association between rural location and anticipated delay in seeking care in SA supports the findings that despite government efforts to make health care more accessible by expanding the number of health facilities, geography remains an important barrier to equitable access to health care $[27,29]$. Addressing financial and geographic access barriers is complex and will require a cross-sectoral approach.

Facility waiting times are another important dimension of access to health care [30] and long waiting time have been reported and contribute to patient dissatisfaction [22, 23, 31, 32]. Relatively few of our participants (13.3\%) felt that long health facility waiting times would be a barrier to seek care for a possible breast or cervical cancer symptom. It is possible that waiting times differ by levels of health care. Our study enquired about accessing primary health care facilities, whereas the other studies [22, 31, 32] were conducted at secondary and tertiary facilities. Research measuring the facility waiting times at the various levels of health care (primary, secondary and tertiary) in LMIC settings will be important in identifying specific bottlenecks in the pathway to cancer care and serve as points for future interventions to improve timely access to care.

We found important differences in the types of barriers reported in our LMIC settings compared to studies conducted in HIC settings [25, 26, 33]. For example, in the UK, concern about wasting a provider's time has been reported as an important barrier to accessing care (30\% Niksic et al [26], $19.5 \%$ Moffat et al [25]), whereas only 3.2\% of women in our study felt this would be a barrier. In a study on anticipated help-seeking for cancer symptoms among ethnic minority groups in the UK, Waller et al [33] found that for more than a third of participants felt that being too embarrassed would be a reason to delay seeking care [33]. In contrast, less than $10 \%$ of our participants felt that this would be a barrier to seeking care. These difference highlights the importance of local studies in informing intervention development.

Most women in our study reported that they would discuss a symptom they considered serious with a family member or friend. This underscores the importance of community level interventions that support women to seek care. Traditional healers are an important part of the health system in Africa [11, 13, 28, 34]. We found that few women $(<10 \%)$ anticipated seeking care from a traditional healer for a possible 
breast or cervical cancer symptom. This low anticipated use could reflect a decline in use of traditional healers, reluctance to disclose use of traditional medicine or that traditional healers might only be visited later in the care pathway. Further qualitative studies could shed light on this.

We found no association between socio-economic status as measured by the asset index and anticipated delay in Uganda, but in SA women with more assets were more likely to delay in seeking care for possible symptoms. Although most studies have found that lower socioeconomic status is associated with greater patient delay in seeking care [11,35], some studies have either reported no association or greater delays in higher compared to lower socio-economic groups [16, 36, 37]. For example, Poum et al [37] found that patient delay for breast cancer symptoms was significantly associated with higher family income [37]. These findings highlight the importance of interventions targeting all income groups. In addition, further research on why SA women with more assets are likely to delay seeking care will be helpful in designing interventions to promote timely care seeking.

Similar to other international studies, we found that greater breast cancer risk factor knowledge was associated with more timely help-seeking behaviour in SA [33, 35]. However, in Uganda, women with higher breast cancer risk factor knowledge were more likely to delay seeking care. Interestingly, in Uganda, endorsement of lay beliefs was associated with more timely help-seeking behaviour. Further qualitative research could shed light on the interplay between risk factor knowledge, endorsement of lay beliefs and anticipated delay in seeking care.

A key strength of our study was that data collection interviews were conducted in the participants home language using a validated questionnaire [14]. Our study also had limitations, as it was hypothetical in nature and responses to a hypothetical symptom may not translate into real-life behaviour. A prospective study design in which help-seeking behaviour for symptoms is investigated as it occurs could be more insightful and less biased. However, prospective designs can have drawbacks, such as the large sample size and resources required for such a study [38]. In our study, selection of sites was directed at bringing forth the uniqueness of each context. While our study offers distinctive SSA perspectives, the four sites are not representative at a country level. Any interpretation and use of results should take this into consideration. We acknowledge that our study focused on barriers and anticipated care-seeking when a symptom is identified as being serious; however, delay in seeking care could also be due to not recognising a symptom.

\section{Conclusion}

We identified factors affecting anticipated help-seeking for possible breast and cervical cancer symptoms. Interventions that improve community level cancer knowledge and highlight the importance of prompt help-seeking for possible symptoms are important to promote timely care seeking. In addition, addressing financial barriers by reducing transport and clinic costs and tackling geographical inequities in access to care could support women in seeking timely care for possible symptoms.

\section{Conflicts of interest}

The authors declare that they have no conflict of interest.

\section{Acknowledgments}

The authors thank the study participants for sharing their knowledge and experiences; Dr Jennifer Githaiga for her commitment, enthusiasm and support throughout the project; the PAC members, field staff and community liaison managers for their support in data collection; Chukwudi Nnaji for assistance with formatting; the Outcome Registry Intervention and Operation Network team, who developed and hosted the electronic surveys on their platform. FMW is a Director and SES is a co-investigator of the multi-institutional CanTest Collaborative, which is funded by Cancer Research UK (C8640/A23385). 


\section{Funding statement}

Research reported in this article was jointly supported by the Cancer Association of South Africa, the University of Cape Town and the SA Medical Research Council with funds received from the SA National Department of Health, GlaxoSmithKline Africa Non-Communicable Disease Open Lab (via a supporting grant Project Number: 023), the UK Medical Research Council (via the Newton Fund). Authors retained control of the final content of the publication. The funders had no role in study design, data collection and analysis, decision to publish or preparation of the manuscript.

\section{Authors' contributions}

JM, FMW, SES and ADM conceptualised and designed the study.

JM, ADM and DC oversaw data collection.

DC curated the data.

DC and JM conducted the quantitative analysis and all authors reviewed the study results.

JM prepared the first draft, incorporated revisions and prepared the final draft. All the authors reviewed drafts and approved the final manuscript.

\section{References}

1. Bray F, Ferlay J, and Soerjomataram I, et al (2018) Global cancer statistics 2018: GLOBOCAN estimates of incidence and mortality worldwide for 36 cancers in 185 countries CA Cancer J Clin 68(6) 394-424 https://doi.org/10.3322/caac.21492 PMID: 30207593

2. The Lancet (2018) GLOBOCAN 2018: counting the toll of cancer Lancet 392(10152) 985 https://doi.org/10.1016/S01406736(18)32252-9 PMID: 30264708

3. Ferlay J, Ervik M, and Lam F, et al (2018). Global Cancer Observatory: Cancer Today. Lyon, France: International Agency for Research on Cancer [https://gco.iarc.fr/today] Date accessed: 8/07/20

4. World Health Organization (WHO) (2020) WHO Report on Cancer: Setting Priorities, Investing Wisely and Providing Care for All (Geneva: World Health Organization) Licence: CC BY-NC-SA 3.0 IGO

5. Joko-Fru WY, Miranda-Filho A, and Soerjomataram I, et al (2020) Breast cancer survival in sub-Saharan Africa by age, stage at diagnosis and human development index: a population-based registry study IJC 146(5) 1208-1218

6. Sengayi-Muchengeti M, Joko-Fru WY, and Miranda-Filho A, et al (2020) Cervical cancer survival in sub-Saharan Africa by age, stage at diagnosis and Human Development Index: A population-based registry study IJC 147(11) 3037-3048

7. Cancer Research UK (CRUK) (2020) Statistics by cancer type [https://www.cancerresearchuk.org/health-professional/cancer-statistics/ statistics-by-cancer-type] Date accessed: 8/10/20

8. Scott SE, Walter FM, and Webster A, et al (2013) The model of pathways to treatment: conceptualization and integration with existing theory BrJ Health Psychol 18(1) 45-65 https://doi.org/10.1111/j.2044-8287.2012.02077.x

9. Walter F, Webster A, and Scott S, et al (2012) The Andersen model of total patient delay: a systematic review of its application in cancer diagnosis J Health Serv Res Policy 17(2) 110-118 https://doi.org/10.1258/jhsrp.2011.010113 PMCID: 3336942 
10. Moodley J, Constant D, and Mwaka AD, et al (2020) Mapping awareness of breast and cervical cancer risk factors, symptoms and lay beliefs in Uganda and South Africa PLoS One 15(10) e0240788 https://doi.org/10.1371/journal.pone.0240788 PMID: 33091035 PMCID: 7580973

11. Akuoko CP, Armah E, and Sarpong T, et al (2017) Barriers to early presentation and diagnosis of breast cancer among African women living in sub-Saharan Africa PLoS One 12(2) e0171024 https://doi.org/10.1371/journal.pone.0171024 PMID: 28192444 PMCID: 5305236

12. McKenzie F, Zietsman A, and Galukande M, et al (2018) Drivers of advanced stage at breast cancer diagnosis in the multicountry African breast cancer - disparities in outcomes (ABC-DO) study IJC 142(8) 1568-1579

13. Pace LE, Mpunga T, and Hategekimana V, et al (2015) Delays in breast cancer presentation and diagnosis at two rural cancer referral centers in Rwanda Oncologist 20(7) 780-788 https://doi.org/10.1634/theoncologist.2014-0493 PMID: 26032138 PMCID: 4492236

14. Moodley J, Scott SE, and Mwaka AD, et al (2019) Development and validation of the African Women Awareness of CANcer (AWACAN) tool for breast and cervical cancer PLoS One 14(8) e0220545 https://doi.org/10.1371/journal.pone.0220545 PMID: 31386684 PMCID: 6684059

15. Rayne S, Schnippel K, and Kruger D, et al (2019) Delay to diagnosis and breast cancer stage in an urban South African breast clinic $S$ Afr Med 109(3) 159-163 https://doi.org/10.7196/SAMJ.2019.v109i3.13283

16. Moodley J, Cairncross L, and Naiker T, et al (2018) From symptom discovery to treatment - women's pathways to breast cancer care: a cross-sectional study BMC Cancer 18(1) 312 https://doi.org/10.1186/s12885-018-4219-7 PMID: 29562894 PMCID: 5863383

17. Rudd P, Gorman D, and Meja S, et al (2017) Cervical cancer in southern Malawi: A prospective analysis of presentation, management, and outcomes Malawi Med J 29(2) 124-129 https://doi.org/10.4314/mmj.v29i2.9 PMID: 28955419 PMCID: 5610282

18. McCutchan GM, Wood F, and Edwards A, et al (2015) Influences of cancer symptom knowledge, beliefs and barriers on cancer symptom presentation in relation to socioeconomic deprivation: a systematic review BMC Cancer 151000 https://doi.org/10.1186/s12885015-1972-8 PMID: 26698112 PMCID: 4688960

19. Andersen BL and Cacioppo JT (1995) Delay in seeking a cancer diagnosis: delay stages and psychophysiological comparison processes Br J Soc Psychol 34 (Pt 1) 33-52 https://doi.org/10.1111/j.2044-8309.1995.tb01047.x PMID: 7735731

20. International Conference on Primary Health Care (1978) Declaration of Alma-Ata WHO Chron 32(11) 428-430 PMID: 11643481. PMID: 11643481

21. World Health Organization (WHO) Declaration of Astana (Geneva: World Health Organization) [https://www.who.int/primary-health/ conference-phc/declaration] Date accessed: 16/08/20

22. Moodley J, Cairncross L, and Naiker T, et al (2016) Understanding pathways to breast cancer diagnosis among women in the Western Cape Province, South Africa: a qualitative study BMJ Open 6(1) e009905 https://doi.org/10.1136/bmjopen-2015-009905 PMID: 26729392 PMCID: 4716174

23. Getachew S, Tesfaw A, and Kaba M, et al (2020) Perceived barriers to early diagnosis of breast Cancer in south and southwestern Ethiopia: a qualitative study BMC Women's Health 20(1) 38 https://doi.org/10.1186/s12905-020-00909-7 PMID: 32103774 PMCID: 7045514

24. Binka C, Nyarko SH, and Awusabo-Asare K, et al (2019) Barriers to the uptake of cervical cancer screening and treatment among rural women in Ghana Biomed Res Int 20196320938 https://doi.org/10.1155/2019/6320938 PMID: 31781631 PMCID: 6874950

25. Moffat J, Hinchliffe R, and Ironmonger L, et al (2016) Identifying anticipated barriers to help-seeking to promote earlier diagnosis of cancer in Great Britain Public Health 141 120-125 https://doi.org/10.1016/j.puhe.2016.08.012 PMID: 27931986 PMCID: 5157686 
26. Niksic M, Rachet B, and Warburton FG, et al (2015) Cancer symptom awareness and barriers to symptomatic presentation in Englandare we clear on cancer? Br J Cancer 113(3) 533-542 https://doi.org/10.1038/bjc.2015.164 PMID: 26125450 PMCID: 4522620

27. Burger R and Christian C (2020) Access to health care in post-apartheid South Africa: availability, affordability, acceptability Health Econ Policy Law 15(1) 43-55 https://doi.org/10.1017/S1744133118000300

28. Mwaka AD, Garimoi CO, and Were EM, et al (2016) Social, demographic and healthcare factors associated with stage at diagnosis of cervical cancer: cross-sectional study in a tertiary hospital in Northern Uganda BMJ Open 6(1) e007690 https://doi.org/10.1136/bmjopen-2015-007690 PMID: 26801459 PMCID: 4735146

29. Dickens C, Joffe M, and Jacobson J, et al (2014) Stage at breast cancer diagnosis and distance from diagnostic hospital in a periurban setting: a South African public hospital case series of over 1,000 women IJC 135(9) 2173-2182

30. Thiede M, Akweongo P, and Mclntyre D (2007) Exploring the dimensions of access In D Mclntyre and G Mooney The economics of health equity eds 1st edn (New York: Cambridge University Press) pp.103-123 https://doi.org/10.1017/CBO9780511544460.007

31. Mwaka AD, Okello ES, and Wabinga H, et al (2015) Symptomatic presentation with cervical cancer in Uganda: a qualitative study assessing the pathways to diagnosis in a low-income country BMC Women's Health 1515 https://doi.org/10.1186/s12905-015-0167-4 PMID: 25783641 PMCID: 4337099

32. Unger-Saldaña K, Ventosa-Santaulària D, and Miranda A, et al (2018) Barriers and explanatory mechanisms of delays in the patient and diagnosis intervals of care for breast cancer in Mexico Oncologist 23(4) 440-453 https://doi.org/10.1634/theoncologist.2017-0431 PMCID: 5896704

33. Waller J, Robb K, and Stubbings S, et al (2009) Awareness of cancer symptoms and anticipated help seeking among ethnic minority groups in England BrJ Cancer 101(Suppl 2) S24-S30 https://doi.org/10.1038/sj.bjc.6605387 PMID: 19956159 PMCID: 2790709

34. Mokgobi MG (2014) Understanding traditional African healing Afr J Phys Health Educ Recreat Dance 20(Suppl 2) 24-34 PMID: 26594664 PMCID: 4651463

35. Khakbazan Z, Taghipour A, and Latifnejad Roudsari R, et al (2014) Help seeking behavior of women with self-discovered breast cancer symptoms: a meta-ethnographic synthesis of patient delay PLoS One 9(12) e110262 https://doi.org/10.1371/journal.pone.0110262 PMID: 25470732 PMCID: 4254513

36. Bhatia RK, Rayne S, and Rate W, et al (2018) Patient factors associated with delays in obtaining cancer care in Botswana J Glob Oncol 4 1-13

37. Poum A, Promthet S, and Duffy SW, et al (2014) Factors associated with delayed diagnosis of breast cancer in northeast Thailand J Epidemiol 24(2) 102-108 https://doi.org/10.2188/jea.JE20130090 PMCID: 3983282

38. Scott S and Walter F (2010) Studying help-seeking for symptoms: the challenges of methods and models Soc Personal Psych Compass 4(8) 531-547 https://doi.org/10.1111/j.1751-9004.2010.00287.x 